\title{
Stable Subloop Behavior in Ferroelectric Si-Doped
}

\section{$\mathrm{HfO}_{2}$}

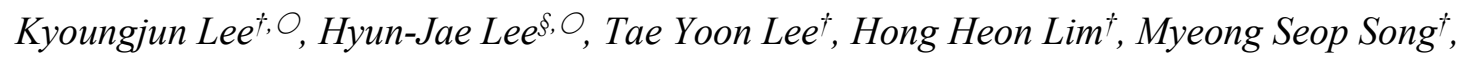

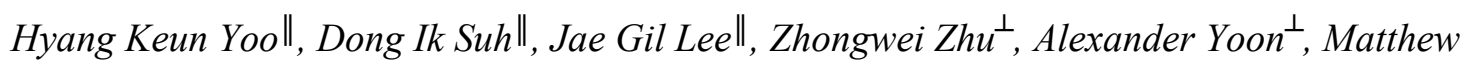

R. MacDonald ${ }^{\nabla}$, Xinjian Lei ${ }^{\nabla}$, Kunwoo Park* ${ }^{*}$, Jungwon Park*\#, Jun Hee Lee ${ }^{*, \xi}$ and Seung

\section{Chul Chae*,}

†Department of Physics Education, ${ }^{\star}$ School of Chemical and Biological Engineering, Institute of Chemical Process, Seoul National University, Seoul 08826, Korea

$\S$ School of Energy and Chemical Engineering, Ulsan National Institute of Science and Technology (UNIST), Ulsan 44919 (Korea)

"SK Hynix Inc., Icheon-si, Gyeonggi-do 17336, Korea

${ }^{\perp}$ Lam Research Corp., Fremont, CA 94538, USA

${ }^{\nabla}$ Versum Materials, Inc., Carlsbad, CA 92011, USA

${ }^{\#}$ Center for Nanoparticle Research, Institute for Basic Science (IBS), Seoul 08826, Korea 


\section{Corresponding Author}

*E-mail: $\underline{\text { scchae@snu.ac.kr }}$

*E-mail: junhee@,unist.ac.kr 


\section{Effects of Si-doping concentration}

To optimize the ferroelectric properties, we varied Si-doping concentration from $2.3 \mathrm{~mol} \%$ to $5.7 \mathrm{~mol} \%$. The hysteresis measurements were conducted after the wake-up process, by inducing 10,000 voltage pulse cycles of $3 \mathrm{~V}$ at a frequency of $10 \mathrm{kHz}$. Figure S1a-c show the measured ferroelectric hysteresis at different temperature. Measured remnant polarization value at room temperature of $2.3 \mathrm{~mol} \%, 4.2 \mathrm{~mol} \%$ and $5.7 \mathrm{~mol} \%$ was about $16.6 \mu \mathrm{C} / \mathrm{cm}^{2}, 28.2$ $\mu \mathrm{C} / \mathrm{cm}^{2}$ and $2.8 \mu \mathrm{C} / \mathrm{cm}^{2}$, respectively. Figure S1d shows the temperature dependence of the coercive field with different Si-doping concentration. The results were fitted by the same equation (5) in main manuscript. The fitted critical volume for nucleation $\left(\mathrm{V}^{*}\right)$ of $2.3 \mathrm{~mol} \%$, $4.2 \mathrm{~mol} \%$, and $5.7 \mathrm{~mol} \%$ samples were $6.6 \times 10^{-27} \mathrm{~m}^{3}, 4.0 \times 10^{-27} \mathrm{~m}^{3}$ and $3.8 \times 10^{-27} \mathrm{~m}^{3}$, respectively. The fitted energy barrier for unit volume (WB) of $2.3 \mathrm{~mol} \%, 4.2 \mathrm{~mol} \%$ and 5.7 mol\% samples were $3.7 \times 10^{26} \mathrm{eV} / \mathrm{m}^{3}, 4.1 \times 10^{26} \mathrm{eV} / \mathrm{m}^{3}$ and $2.3 \times 10^{26} \mathrm{eV} / \mathrm{m}^{3}$, respectively. Compared to conventional perovskite materials ferroelectric $\mathrm{HfO}_{2}$ exhibited small critical volume for nucleation with large energy barrier for unit volume. Considering the large polarization value, small critical volume for nucleation and large energy barrier for unit volume, we chose the $4.2 \mathrm{~mol} \% \mathrm{Si}$-doped $\mathrm{HfO}_{2}$ films for the detailed analysis in the main manuscript.

\section{Grain size estimation}

Size of domains was estimated from high resolution transmission electron microscope (HRTEM) images. The lamella for HRTEM observation was produced by focused ion beam 
(FIB). To achieve high resolution, nano mill and TEM imaging were repeated on the lamella until the optimal thickness is attained for HRTEM. The final 20-nm thick lamella was characterized by using electron energy loss spectroscopy (EELS).

As-prepared lamella sample of $\mathrm{Si}$-doped $\mathrm{HfO}_{2}$ was investigated by using $\mathrm{C}_{\mathrm{s}}$ corrected TEM and atomic resolution images were obtained as shown in Figure S2. HRTEM images with clear identification of lattice structures enabled the grain boundary characterization by strain mapping. Geometric phase analysis (GPA) was utilized to obtain strain maps ${ }^{1}$ (the inset of Figure S2) which display grain boundaries between grains comprising $\mathrm{HfO}_{2}$ film as well as the interface between $\mathrm{HfO}_{2}$ film and TiN electrodes. The HRTEM images were consecutively obtained throughout the entire lamella specimen to accurately measure the size of grains in sufficiently high magnification for atomic resolution.

Based on the grain map from atomic resolution TEM images and their GPA analysis, projected area of grain sizes was estimated about $240 \mathrm{~nm}^{2}$ on average. Since the thickness of $\mathrm{HfO}_{2}$ layer is $8 \mathrm{~nm}$ consistently over the entire film, the width of the grains can be estimated around 30 $\mathrm{nm}$.

3. Synaptic behavior of polarization

To study the synaptic characteristics of polarization value, we measured the remnant polarization value after applying the pulses sequences as shown in Figure S1. Pulse scheme 1 consists of identical voltage pulses with height of $+3.0 \mathrm{~V}$ (potentiation), $-3.2 \mathrm{~V}$ (depression) and width of 600 ns. Pulse scheme 2 consists of pulses with increasing width increment of 50 ns with fixed height of $+3.0 \mathrm{~V}$ (potentiation) and $-3.2 \mathrm{~V}$ (depression). Pulse scheme 3 consists of pulses with increasing height increment of $0.1 \mathrm{~V}$ with fixed width of $600 \mathrm{~ns}$. To read the 
remnant polarization states after applying each voltage pulses sequence, voltage pulses with height and frequency of $3 \mathrm{~V}$ and $2 \mathrm{kHz}$, respectively, were applied for the read and reset process. Figure S3d,e show the measured potentiation and depression behavior of programmed polarization. In the case of pulse scheme 1, the polarization value was saturated before reaching the maximum/minimum saturation value in both potentiation and depression cases. In the case of pulse scheme 2 , the polarization value was fully saturated in both potentiation and depression cases. In the case of pulse scheme 3, the polarization value was fully saturated for potentiation case while the polarization value did not saturate for depression case. It can be attributed to the poly-crystallinity of $\mathrm{HfO}_{2}$ thin film. The randomly oriented grains require different coercive voltages for the full saturation of polarization. Thus pulse scheme 1 where the voltage pulse and width were fixed cannot induce the full saturation. ${ }^{2}$

\section{High temperature retention properties}

To check the robust stability of each intermediate polarization state even at high temperature, we measured the time evolution of $\Delta \mathrm{P}(\mathrm{t}) / \Delta \mathrm{P}_{0}$ at $125^{\circ} \mathrm{C}$. Figure $\mathrm{S} 4$ shows the retention of an intermediate polarization state with applying pulses with height and width of $3 \mathrm{~V}$ and $2 \mu \mathrm{s}$, respectively. The results exhibited only a little difference with respect to the $1 \mathrm{~s}$ after 10,000 s. For the detailed analysis, the polarization loss was fitted with the stretched exponential decay as below.

$\frac{\Delta \mathrm{P}(\mathrm{t})}{\Delta P_{0}}=\mathrm{A} \times \exp \left(-\left(\frac{\mathrm{t}}{t_{0}}\right)^{\beta}\right)$ 
The fitted $t_{0}$ and stretched exponent $\beta$ were $3.3 \times 10^{15} \mathrm{~s}$ and 0.12 , respectively.

\section{Details of switching dynamics}

To elucidate the time and voltage dependences of ferroelectric switching, we measured the switched polarization value $\Delta \mathrm{P}$ after applying the voltage pulses, as shown in Figure S5a. The pristine sample was polled negatively by applying a triangular pulse of $-3 \mathrm{~V}$ at $2 \mathrm{kHz}$ to the top electrode. Subsequently, square-shaped voltage pulses over the range of $0.9-3 \mathrm{~V}$ and width of $100 \mathrm{~ns}$ to $1 \mathrm{~ms}$ were applied to the top electrode. To read the $\Delta \mathrm{P}$ value, we induced polling twice at $3 \mathrm{~V}$ and $2 \mathrm{kHz}$; the former pulse included the switching current and non-switching current, while the latter pulse included only the non-switching current. Spontaneous polarization $2 \mathrm{P}_{\mathrm{s}}$ was measured by the reading pulse twice, without square-shaped voltage pulses at the negatively polled sample. The $\Delta \mathrm{P}$ value was calculated as the difference between the spontaneous polarization value $2 \mathrm{P}_{\mathrm{s}}$ and the ferroelectric switching component in the former reading pulse. Figure $\mathrm{S} 1 \mathrm{~b}$ shows the Lorentzian distribution of the fitting function at different voltage pulse heights. ${ }^{3}$ The switching activation field in Figure $5 \mathrm{~b}$ of the main manuscript was estimated from the peak position in each Lorentzian distribution.

\section{Details of theoretical calculations}

For the theoretical estimation of the energy difference, we conducted first-principles calculations based on density functional theory using Vienna Ab initio simulation package code (VASP Software GmBH, Vienna, Austria). ${ }^{4-7}$ We adopted the generalized gradient approximation method for describing the exchange correlation functional and pseudopotentials 
generated under the projector-augmented plane wave scheme by Perdew, Burke, and Ernzerhof. ${ }^{8,9}$ A $4 \times 4 \times 4$ k-point grid was chosen for the Monkhorst-Pack (MP) method involving integration within the Brillouin zone..$^{10}$ The energy cut-off for the plane wave basis was set to $500 \mathrm{eV}$, and the force criterion for structure optimization was $0.01 \mathrm{eV} / \AA$. The unit cell contained four formula units: orthorhombic $\mathrm{HfO}_{2}$ with $a=5.2733(7) \AA, b=5.0551(7) \AA$, and $c=5.0851(9) \AA$, and tetragonal $\mathrm{PbTiO}_{3}(\mathrm{PTO})$ with $a=3.86$ and $c=4.04$. The Berry phase method was used to calculate the electric polarization. ${ }^{11,12}$ The calculated spontaneous polarizations of $\mathrm{HfO}_{2}$ and PTO were 41 and $60 \mu \mathrm{C} / \mathrm{cm}^{2}$, respectively. Our calculated polarization well matched experimentally and theoretically determined values. ${ }^{13,14}$ The activation barrier for polarization switching was calculated with $1 \times 8 \times 1$ supercells containing a $4 \times 1 \times 4$ k-point grid with 32 formula units of $\mathrm{HfO}_{2}$ and PTO. Figure $\mathrm{S} 6$ a shows the number of ferroelectric dipole flips as a function of the energy per unit dipole flip (i.e., total energy/number of dipoles) landscape in PTO. In the case of a single dipole flip (see Figure 5c in the main manuscript), the energy difference between the energy barrier height and after the dipole flip was small $(0.12 \mathrm{eV})$; the small energy difference indicates that a single dipole flip in PTO is not stable. As the number of ferroelectric dipole flips increased, the energy difference between the barrier height and after the dipole flip increased. Therefore, PTO requires more than two unit cells of ferroelectric dipole flips to stabilize the dipoles after flipping. In the case of $\mathrm{HfO}_{2}$, the barrier height value plateaued at $1.34 \mathrm{eV}$, even in the case of a $1 \times 2 \times 1$ supercell, as shown in Figure S6b. Therefore, the calculated total energy difference of a single ferroelectric dipole flip (see Figure $5 \mathrm{c}$ in the main manuscript) using a $1 \times 8 \times 1$ supercell was appropriate. Figure S6c shows the calculated total energy difference after several ferroelectric 
dipole flips using a $1 \times 10 \times 1$ supercell. The relative total energy difference was less than 0.1 meV/f.u.; this energy difference is considered to be negligible.

7. Details of the Monte Carlo simulations

To elucidate the domain size effects of ferroelectric switching on the reproducibility of intermediate polarization states, we conducted a Monte Carlo simulation using the following Hamiltonian $^{15}$

$$
\mathrm{H}=\sum_{j} \frac{P_{i} \cdot P_{j}-3\left(P_{i} \cdot n\right)\left(P_{j} \cdot n\right)}{r^{3}}-P_{i} \cdot E_{\text {ext }},
$$

where $P_{i}, r, n$, and $E_{\text {ext }}$ represent the ferroelectric dipole moment at lattice site i, the distance between dipoles, the unit vector connecting dipoles, and the external electric field, respectively. The simulation used a defect density of $4 \%$ and assumed that the pinned dipoles in domains were the result of charged defects and/or oxygen vacancies in a polycrystalline ferroelectric $\mathrm{HfO}_{2}$ film ${ }^{16,17}$. In the simulation, we used $\mathrm{K}_{\mathrm{B}} \mathrm{T}=1$, and an external field $E=9.0$ (a. u.). Monte Carlo simulations were performed under the conditions specified in a previous study, ${ }^{18}$ considering the NLS kinetics in a ferroelectric polycrystalline $\mathrm{HfO}_{2}$ thin film. We considered dipole interactions up to the third nearest neighbor for the mean switching time. The relative magnitude of the defect dipole was varied randomly from -1 to 1 .

Figure S7a shows the mesh grid size dependence of the hysteresis loops. For the $10 \times 10$ and $20 \times 20$ cases, irregular hysteresis loops were observed due to the irregular noise associated with back switching and time autocorrelation during the simulation. On the other hand, simulations conducted with mesh grid sizes larger than 50 exhibited clear hysteresis. Therefore, we examined the system size dependence of accessibility to the intermediate polarization states 
with respect to the mesh grid size. Figure S7b shows the MCS dependence of polarization switching dynamics for a mesh grid size of $100 \times 100$, repeated 100 times. 
Figure S1
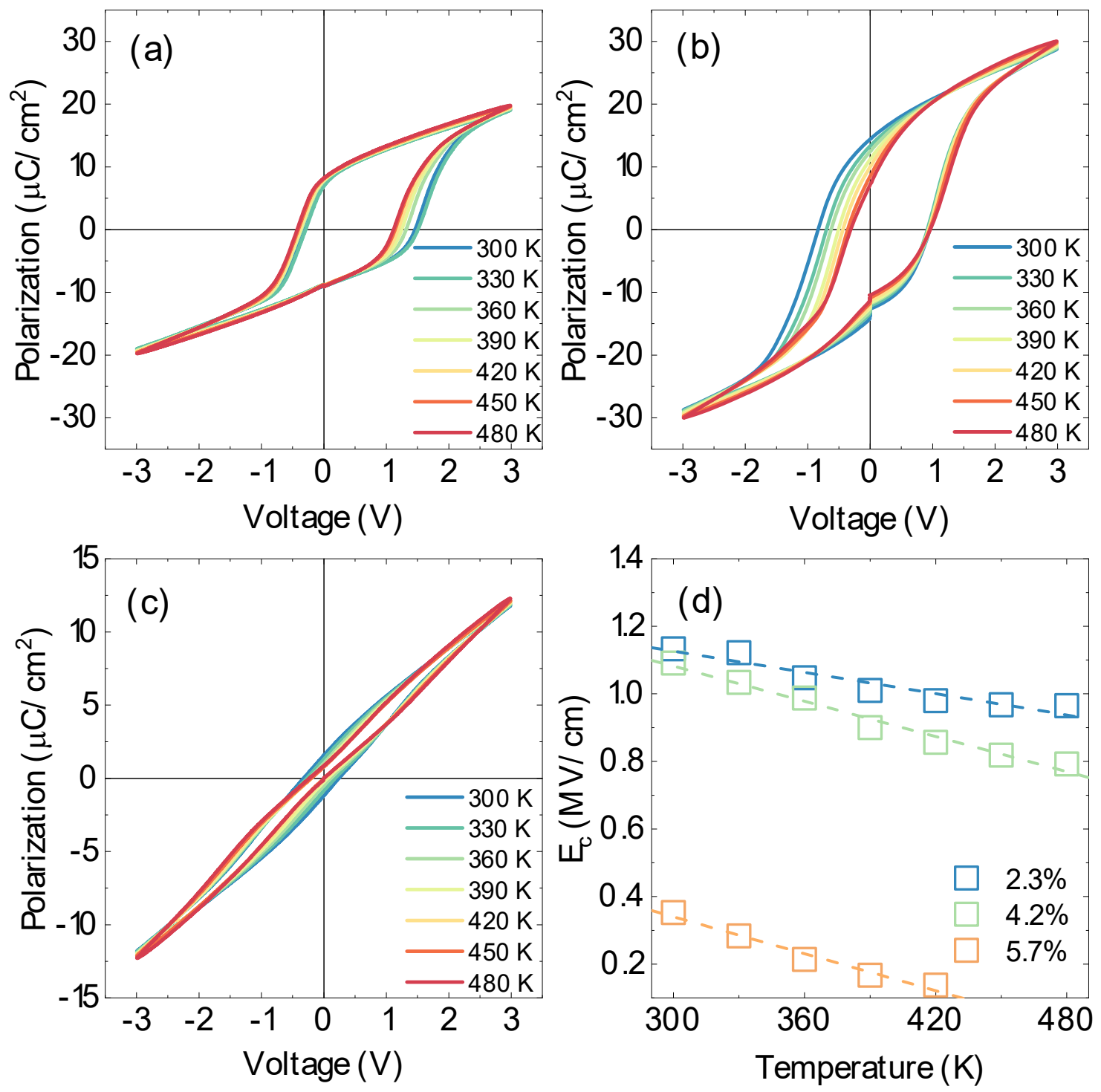

Figure S1. Temperature dependence of ferroelectric hysteresis with different Si doping concentration (a) $2.3 \mathrm{~mol} \%$, (b) $4.2 \mathrm{~mol} \%$ and (c) $5.7 \mathrm{~mol} \%$. (d) Temperature dependence of the coercive filed. 
Figure S2

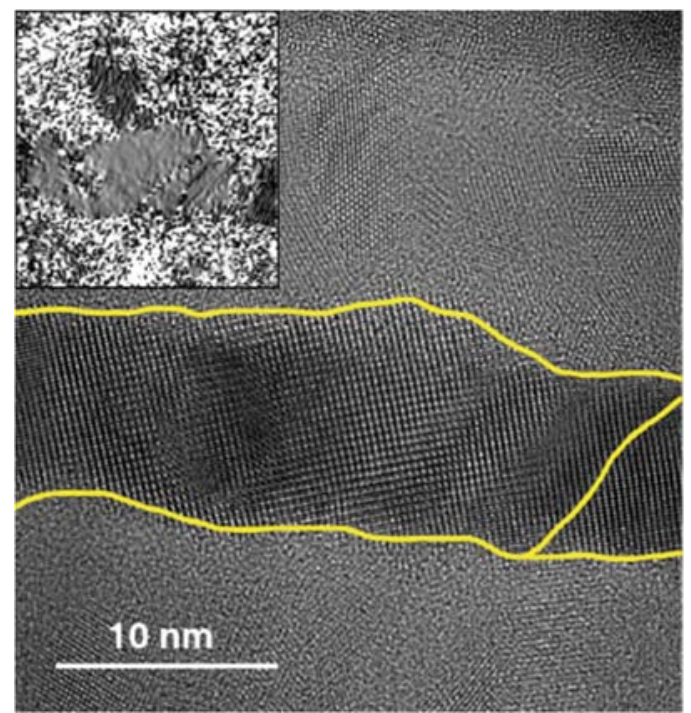

Figure S2. A cross sectional HRTEM image of the Si-doped $\mathrm{HfO}_{2}$ film. The inset is the deformation map used to indicate grain boundaries (yellow line). 
Figure S3

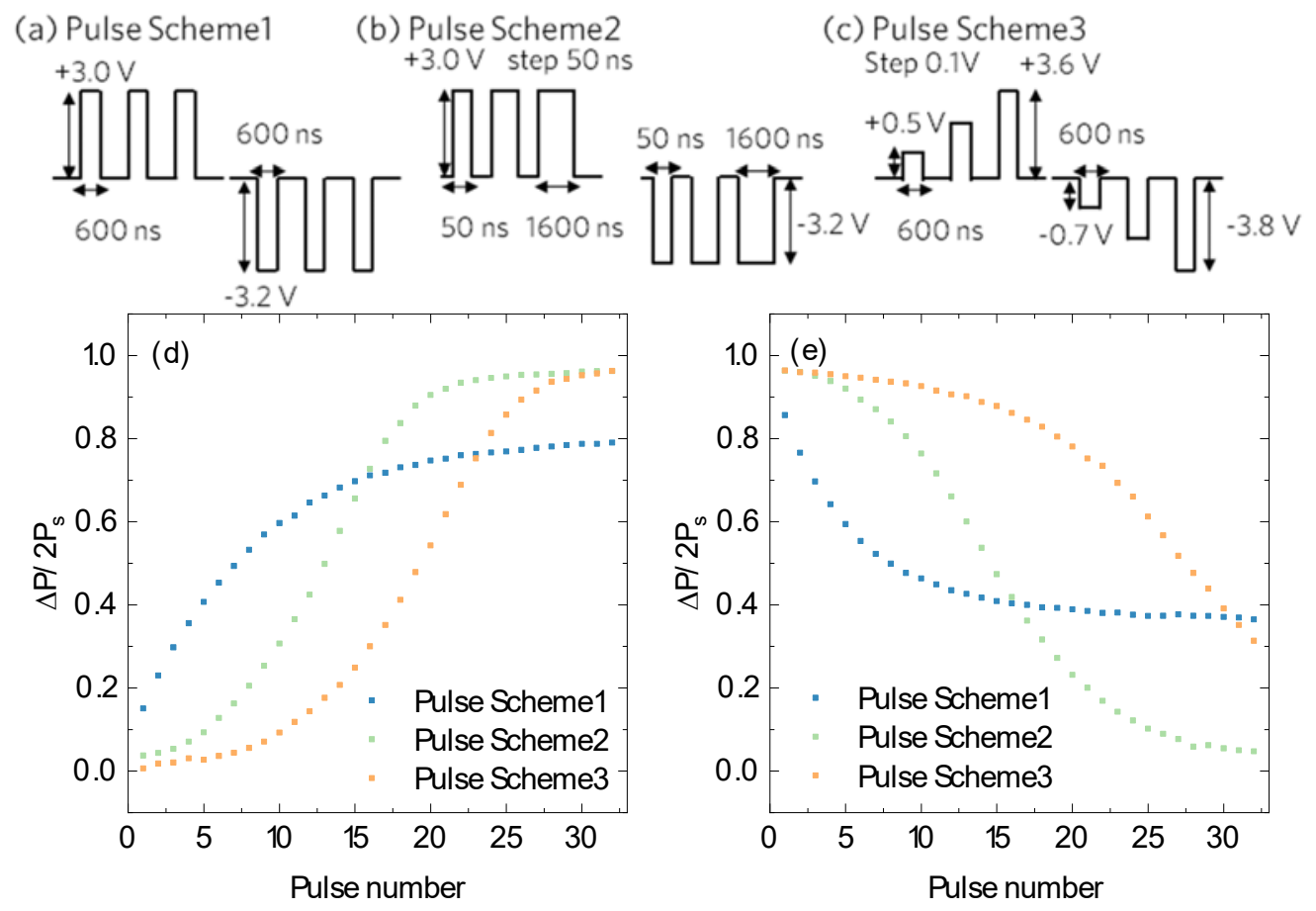

Figure S3. Schematics of various programming pulse schemes with (a) identical pulse width and height (b) increasing pulse width and (c) increasing pulse height for potentiation and depression, respectively. (d) Potentiation and (e) depression behavior of the polarization in Sidoped $\mathrm{HfO}_{2}$ film. 
Figure S4

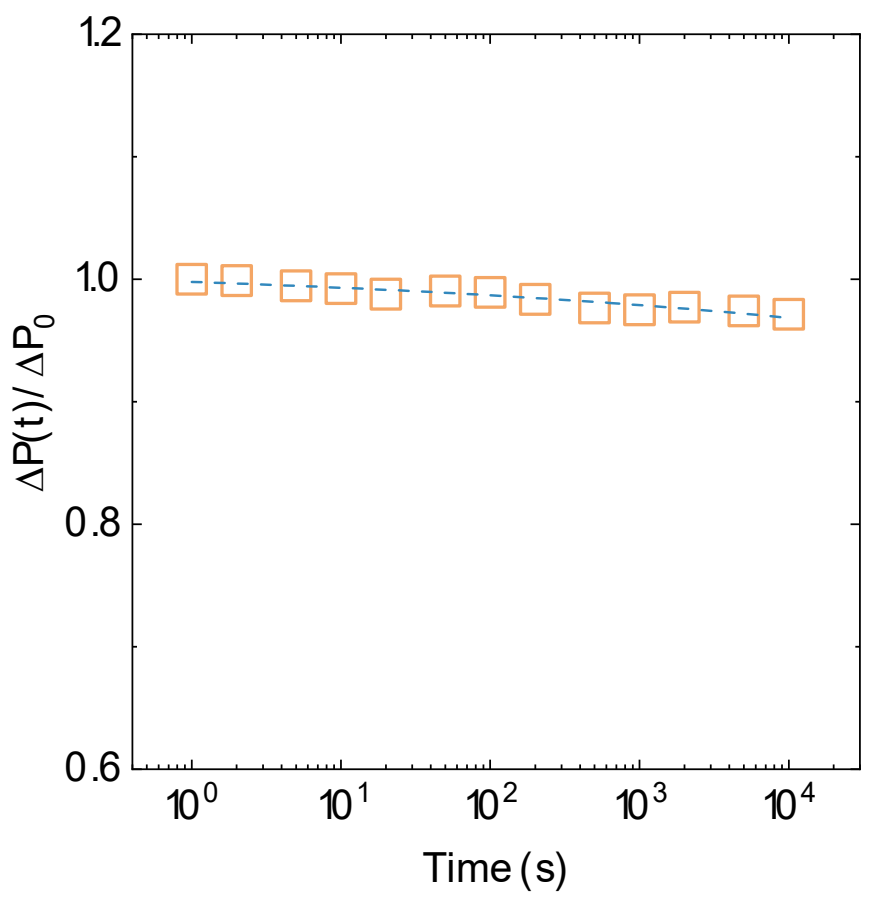

Figure S4. Retention properties measured at $125^{\circ} \mathrm{C}$. The dashed fitting line corresponds to the model fitted with the stretched exponential decay. 
Figure S5
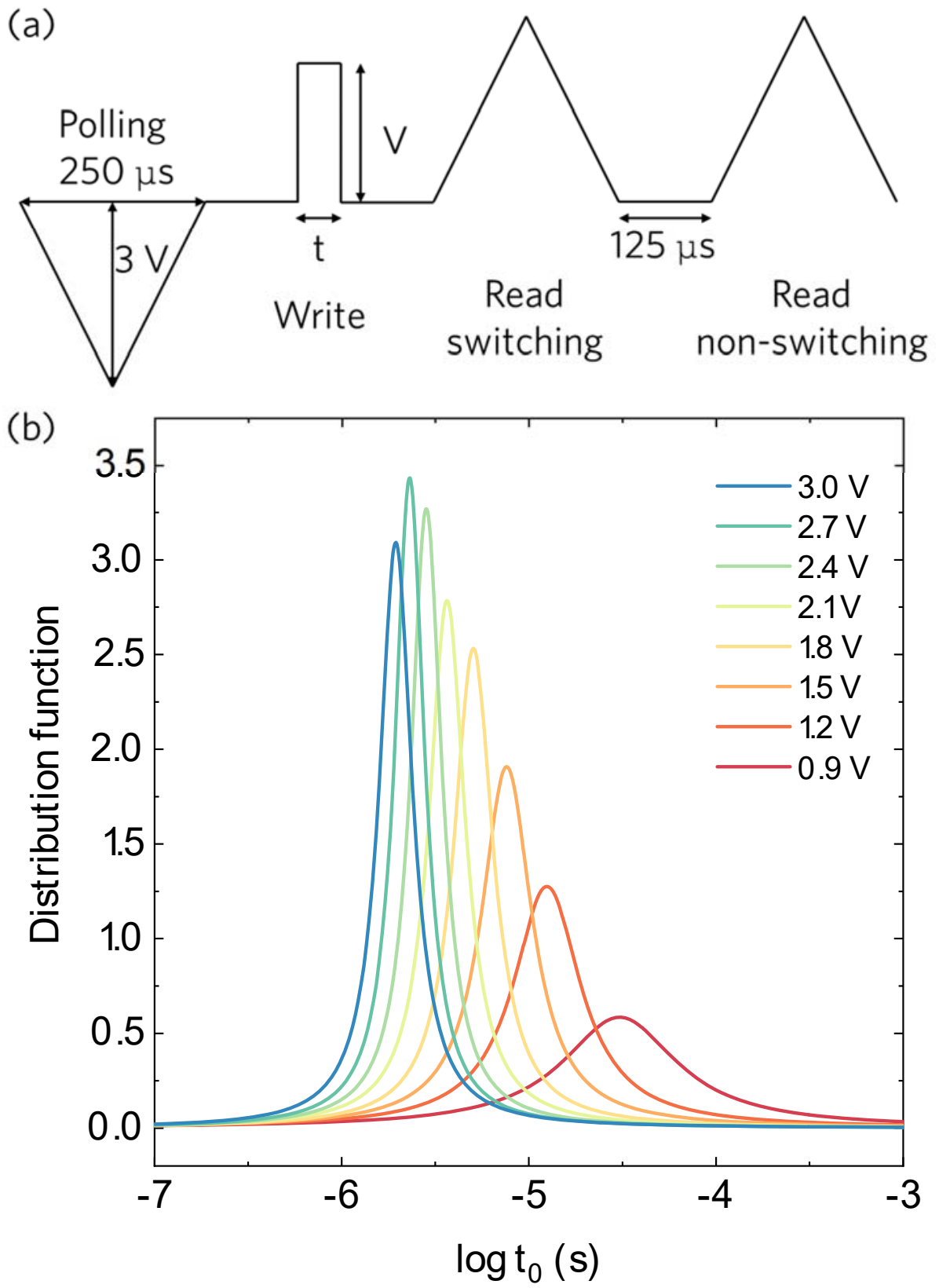

Figure S5. (a) Voltage pulse sequence schematic diagram used to measure the switching dynamics. (b) Lorentzian distribution of fitting function at different voltage pulse heights. 
Figure S6
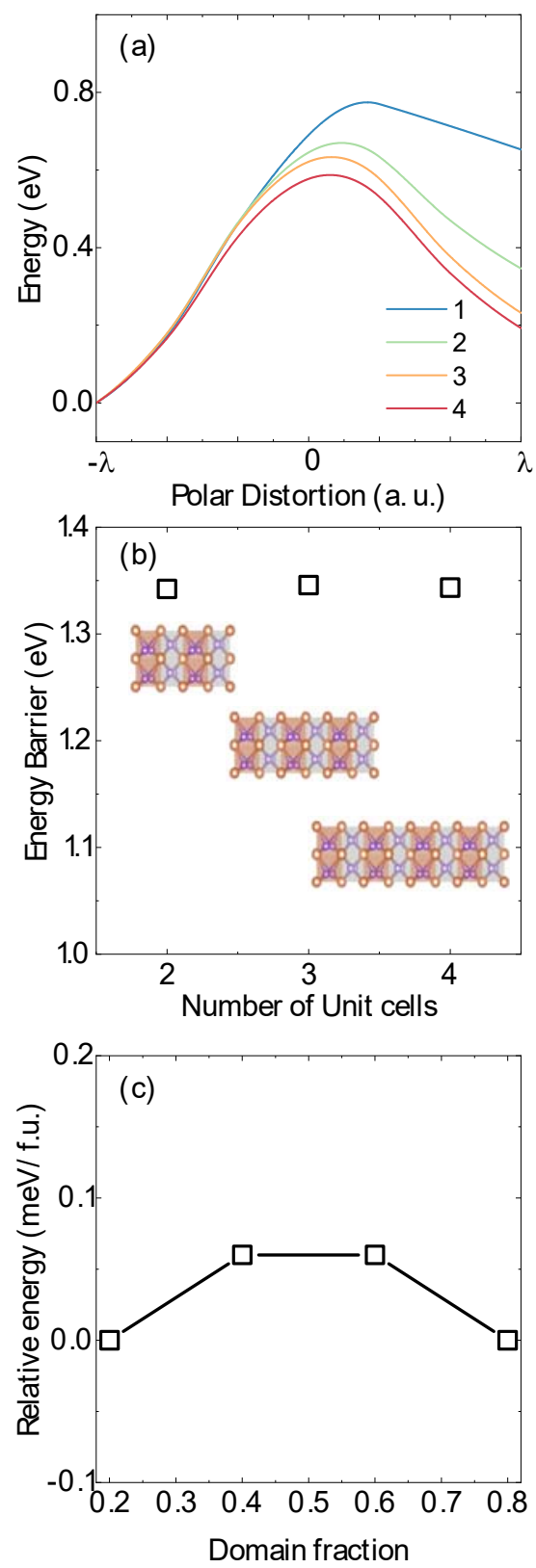

Figure S6. (a) Number of ferroelectric dipole flip dependence of energy landscape in PTO. (b) Supercell size dependence of energy barrier height during a single ferroelectric dipole flip in $\mathrm{HfO}_{2}$. (c) The flip ratio dependence of the total energy in $\mathrm{HfO}_{2}$. 
Figure S7
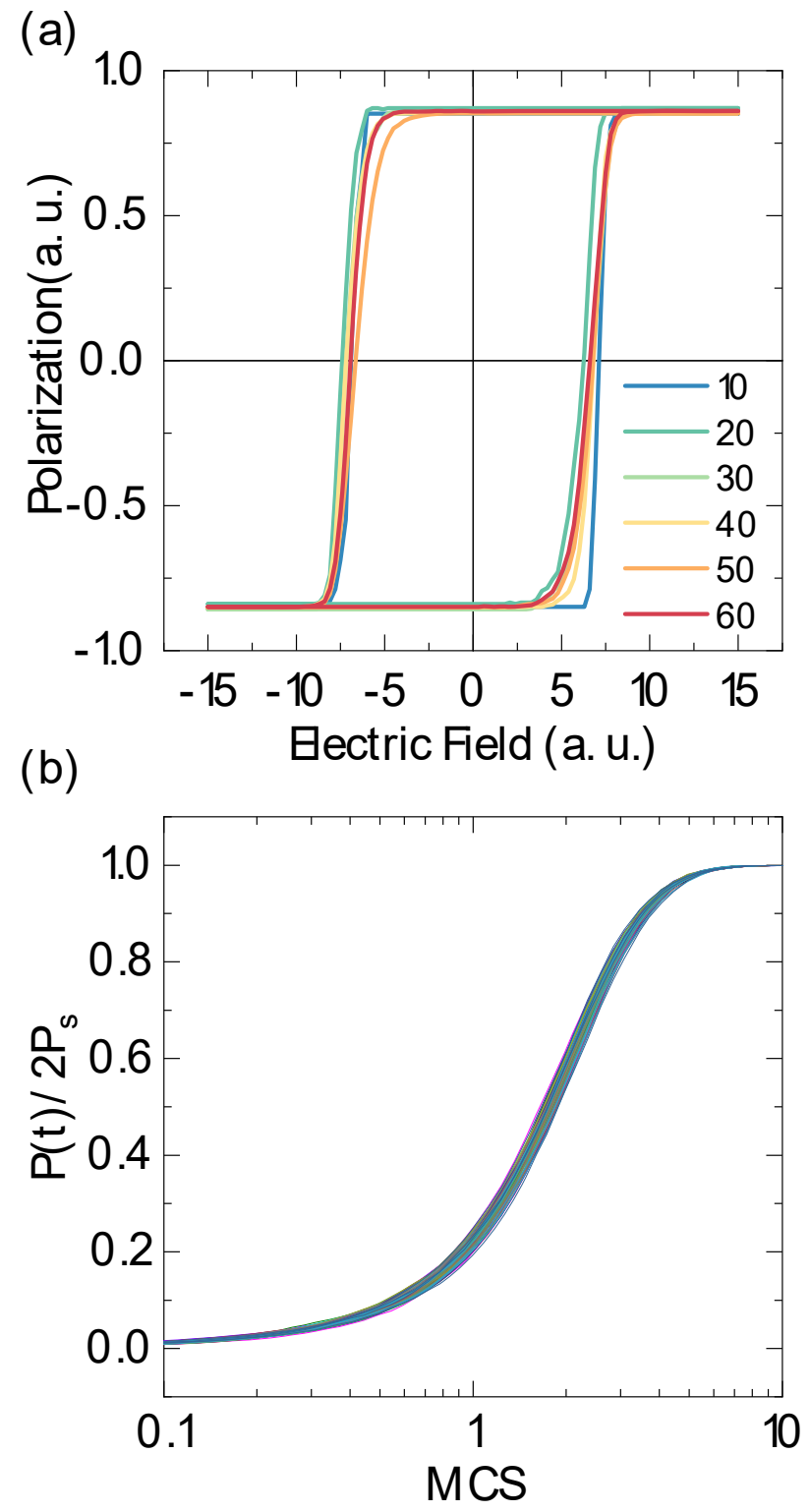

Figure S7. (a) Mesh grid size dependence of hysteresis loops. (b) Repeated polarization reversal behavior (100 times) with a mesh gird size of $100 \times 100$. 


\section{REFERENCES}

(1) Hÿtch, M. J.; Snoeck, E.; Kilaas, R. Quantitative Measurement of Displacement a nd Strain Fields from HREM Micrographs. Ultramicroscopy 1998, 74 (3), 131-146.

(2) Oh, S.; Kim, T.; Kwak, M.; Song, J.; Woo, J.; Jeon, S.; Yoo, I. K.; Hwang, H. $\mathrm{HfZrO}_{\mathrm{x}}$-Based Ferroelectric Synapse Device With 32 Levels of Conductance States for Neuromorphic Applications. IEEE Electron Device Letters 2017, 38 (6), 732-735.

(3) Jo, J. Y.; Han, H. S.; Yoon, J. G.; Song, T. K.; Kim, S. H.; Noh, T. W. Domai n Switching Kinetics in Disordered Ferroelectric Thin Films. Phys. Rev. Lett. 2007, 9 9 (26), 267602.

(4) Kresse, G.; Hafner, J. Ab Initio Molecular Dynamics for Liquid Metals. Phys. Rev . $B$ 1993, 47 (1), 558-561.

(5) Kresse, G.; Furthmüller, J. Efficiency of Ab-Initio Total Energy Calculations for Metals and Semiconductors Using a Plane-Wave Basis Set. Comput. Mater. Sci. 1996, $6(1), 15-50$.

(6) Kresse, G.; Furthmüller, J. Efficient Iterative Schemes for Ab Initio Total-Energy Calculations Using a Plane-Wave Basis Set. Phys. Rev. B 1996, 54 (16), 11169-11186

(7) Kresse, G.; Joubert, D. From Ultrasoft Pseudopotentials to the Projector Augmente d-Wave Method. Phys. Rev. B 1999, 59 (3), 1758-1775.

(8) Blöchl, P. E. Projector Augmented-Wave Method. Phys. Rev. B 1994, 50 (24), 17 953-17979.

(9) Perdew, J. P.; Burke, K.; Ernzerhof, M. Generalized Gradient Approximation Mad e Simple. Phys. Rev. Lett. 1996, 77 (18), 3865-3868. 
(10) Monkhorst, H. J.; Pack, J. D. Special Points for Brillouin-Zone Integrations. Phys . Rev. B 1976, 13 (12), 5188-5192.

(11) King-Smith, R. D.; Vanderbilt, D. Theory of Polarization of Crystalline Solids. $P$ hys. Rev. B 1993, 47 (3), 1651-1654.

(12) Vanderbilt, D.; King-Smith, R. D. Electric Polarization as a Bulk Quantity and It s Relation to Surface Charge. Phys. Rev. B 1993, 48 (7), 4442-4455.

(13) Ederer, C.; Spaldin, N. A. Effect of Epitaxial Strain on the Spontaneous Polariza tion of Thin Film Ferroelectrics. Phys. Rev. Lett. 2005, 95 (25), 257601.

(14) Park, M. H.; Lee, Y. H.; Kim, H. J.; Kim, Y. J.; Moon, T.; Kim, K. D.; Mülle r, J.; Kersch, A.; Schroeder, U.; Mikolajick, T.; Hwang, C. S. Ferroelectricity and An tiferroelectricity of Doped Thin $\mathrm{HfO}_{2}$-Based Films. Adv. Mater. 2015, 27 (11), 1811-1 831.

(15) Wu, Y.-Z.; Yao, D.-L.; Li, Z.-Y. Monte-Carlo Simulation of the Switching Beha vior in Ferroelectrics with Dipolar Defects. Solid State Commun. 2002, 122 (7), 395-4 00.

(16) Schenk, T.; Hoffmann, M.; Ocker, J.; Pešić, M.; Mikolajick, T.; Schroeder, U. C omplex Internal Bias Fields in Ferroelectric Hafnium Oxide. ACS Appl. Mater. Interfa ces 2015, 7 (36), 20224-20233.

(17) Pešić, M.; Fengler, F. P. G.; Larcher, L.; Padovani, A.; Schenk, T.; Grimley, E. D.; Sang, X.; LeBeau, J. M.; Slesazeck, S.; Schroeder, U.; Mikolajick, T. Physical M echanisms behind the Field-Cycling Behavior of $\mathrm{HfO}_{2}$-Based Ferroelectric Capacitors. Adv. Func. Mater. 2016, 26 (25), 4601-4612.

(18) Lee, T. Y.; Lee, K.; Lim, H. H.; Song, M. S.; Yang, S. M.; Yoo, H. K.; Suh, D. I.; Zhu, Z.; Yoon, A.; MacDonald, M. R.; Lei, X.; Jeong, H. Y.; Lee, D.; Park, S-18 
K.; Park, J.; Chae, S. C. Ferroelectric Polarization-Switching Dynamics and Wake-Up Effect in Si-Doped $\mathrm{HfO}_{2}$. ACS Appl. Mater. Interfaces 2018, 11 (3), 3142-3149. 